\title{
PERFIL DE SUSCEPTIBILIDAD EN BACTERIAS COLONIZANTES AISLADAS DE CUCARACHAS EN UN HOSPITAL DE LIMA METROPOLITANA.
}

\section{PROFILE OF SUSCEPTIBILITY IN COLONIZING BACTERIA ISOLATED FROM COCKROACHES IN A HOSPITAL OF METROPOLITAN LIMA.}

\author{
Magna Camones Villanueva, Alfonso Martin Cabello Vilchez
}

\section{Resumen}

Objetivo: Determinar el perfil de susceptibilidad y el Rango de resistencia de bacterias colonizantes aisladas en cucarachas deun Hospital de Lima Metropolitana. Material y Métodos: Estudio descriptivo de corte transversal en los meses de Junio y Julio del año 2015. Se preparó una trampa doméstica con cinta adhesiva para recolectar las cucarachas, se capturaron 100 cucarachas de la especie (Blatella germánica), de diferentes áreas dentro de un Hospital de Lima Metropolitana en un frasco estéril, el aislamiento de bacterias que colonizan en la parte externa de la cucaracha se realizaron por lavado con solución salina al $0,9 \%$, se concentró la muestra, del pellet se realizó tinción de Gram y Ziehl Neelsen. Y se sembraron en medios Agar Sangre y Maconkey, para determinar género y especie por métodos manuales con medios TSI, LIA, CITRATO, UREA y SIM y susceptibilidad y resistencia se realizó por método de difusión o Kirby - Bauer. Los inóculos se sembró en agar Muller Hinton, los discos para Grampositivos (Ampicilina, Oxacilina, Eritromicina, Clindamicina, Vancomicina y cefazolina) y para Gramnegativos (Ciprofloxacino, Trimetroprim + sulfametoxazol, Amikacina, Ceftriaxona, Imipenem y Amoxicilina + Ac. Clavulánico), la lectura comparando con las tablas desarrolladas por NCCLS. Resultados: se aislaron bacterias Grampositivos, el 16\% para Staphylococcus coagulasa - negativa 4\% Streptococcus viridans y bacterias Gramnegativos 24\% Serratiamarcescens, 4\% Acinetobactersp, 36\% Klebsiellapneumoniae, 4\% Klebsiellaoxytoca, 8\% Escherichiacoli y 4\% para Pseudomona sp.Conclusiones: Streptococcus viridans,Klebsiellaoxytoca, y Escherichiacoli fueron los más relevantes y gran de importancia clínica altamente patógenos; causantes de infecciones intrahospitalarias.

PALABRAS CLAVE: Cucarachas, bacterias colonizantes, perfil de susceptibilidad, multidrogoresistente.

\section{Summary}

Objective: To determine the susceptibility profile and resistance range of colonizing bacteria isolated fromof Metropolitan Lima cockroaches. Material and Methods: A cross-sectional descriptive study was carried out in June and July of the year 2015. A domestic trap was prepared with adhesive tape to collect the cockroaches, 100 cockroaches of the species (Blatella germanica) were collected from different áreas within the in a sterile flask, isolation of 
bacteria colonizing the outside of the cockroach were performed by washing with $0.9 \%$ saline solution, the sample was concentrated, the Gram stain and Ziehl Neelsen were stained. And they were planted in Agar Sangre and Maconkey media, to determine genus and species by manual methods with TSI, LIA, CITRATO, UREA and SIM media and susceptibility and resistance was performed by diffusion or Kirby - Bauer method. The inocula were seeded on Muller Hinton agar, Gram-positive disks (Ampicillin, Oxacillin, Erythromycin, Clindamycin, Vancomycin and Cefazolin) and Gram negative (Ciprofloxacin, Trimethoprim + sulfamethoxazole, Amikacin, Ceftriaxone, Imipenem and Amoxicillin + Reading compared to the tables developed by NCCLS. Results: Staphylococcus aureus was isolated from Gram positive bacteria, $16 \%$ for coagulase - negative Staphylococcus 4\% Streptococcus viridans and Gramnegative bacteria 24\% Serratia marcescens, 4\% Acinetobacter sp, 36\% Klebsiella pneumoniae, 4\% Klebsiella oxytoca, 8\% Escherichia coli and 4\% Pseudomonas sp. Conclusions: Streptococcus viridans, Klebsiella oxytoca, and Escherichia coli were the most relevant and large highly pathogenic clinical importance; Causing nosocomial infections.

KEY WORDS: Cockroaches, colonizing bacteria, susceptibility profile, multidrug-resistant.

\section{Introduccion}

Las cucarachas son plagas domésticaspersistentes en áreas urbanas a nivel mundial. Además de las molestias que ocasiona, afecta laeconomía y se considera de gran importanciamédica puesto que alberga y transmite innumerables microorganismospatógenos como: virus, hongos, helmintos ybacterias, responsables de las infecciones nosocomiales; la Blattella germánica, Periplaneta americanason de especie sinantrópica másabundante con una distribución cosmopolita,predominan en las zonas tropicales y templadas, respectivamente, también son una importante fuente de alérgenos, responsables de una serie de enfermedades alérgicas como el asmabronquialy la rinitis alérgica $(1,4,8,9,13,14,17,18,19,20$,$) . Las bacterias que albergan estos$ animales son enteropatógenos,en su mayoría de importancia clínica y epidemiológica como, Escherichia coli (15), Enterobacter spp, Klebsiella spp (23), Pseudomona aeruginosa (24), Acinetobacter baumannii (26), otras bacterias no fermentadoras, Serratia marcescens (27), Shigella spp, Staphylococcus aureus, estreptococos del grupo A (25), Enterococos spp, Bacillus spp(4). Entre estosmicroorganismos existe una alta prevalencia de resistencia a antibióticos y a las insecticidas $(2,7,16,21,22)$. Estos insectos se encuentran en diferentes sectores del hospital, ya que se sienten atraídos por los alimentos, residuos orgánicos y fluidos que se evacúan regularmente en estos lugares.Por lo tanto la introducción de los antibióticos en la terapéutica de las enfermedades infecciosas y su uso generalizado e indiscriminado ha provocado la selección de cepas multidrogoresistentes $(3,11)$.

En un estudio comparativo realizado en los años de 1985 a 1989 en la India, se evaluaron 279 cucarachas de la especie Blatella germánica, se recolectaron159 en un establecimiento de salud grupo de estudio y 120 
alrededor del centro de salud grupo control; demostró presencia de microorganismos patógenos de importancia médica aislados de laparte externa e interna de las cucarachas donde se obtuvo un porcentaje considerable de $99.4 \%$ del grupo de estudio y $94.2 \%$ del grupo control. Asimismo, el $47.1 \%$ fueron bacterias no patógenos en el grupo de estudio y $65.8 \%$ en el grupo control la diferencia otra vez fue estadísticamente significativa (5).

En Taiwán el año 2000 se realizó un estudio a 203 cucarachas (64 Blatella germánica y 139 Periplaneta americana) en 90 hospitales para determinar el rol de estos insectos en la diseminación de bacterias causantes de infecciones intrahospitalarias, se recolectados en áreas clínicas y no clínicas. También se evaluó la resistencia a los antibióticos de las bacterias aisladas del tracto alimentario y de la superficie externa donde se obtuvo elevada prevalencia de bacterias antibiótico resistentes y algunos antibiótico susceptibles. Asimismo de los hospitales estudiados $46.7 \%$ se encuentran infestadas de cucarachas (2).

Dos años después se realizó un estudio de perfil de resistencia antimicrobiana de 103 cucarachas Periplaneta americano, en una institución de salud en Brasil. Se recolectaron de cinco unidades diferentes: las habitaciones, sala de cirugía, enfermería, cafetería y los servicios de nutrición. Entre ellas, 91 dió crecimiento microbiano, de la cual se obtuvieron 126 aislamientos; $71.4 \%$ tenían especies de la familia Enterobacteriaceae, 25.4\% estafilococos coagulasa - negativo y $3.2 \%$ fueron bacilos Gram-negativos. También fueron detectados levaduras y hongos filamentosos, en $97 \%$ de las cucarachas, y el número promedio de los tipos de microorganismos aislados por cucaracha varió de uno a tres (11).

Tres años después en África se realizó un estudio a 234 cucarachas recolectadas en viviendas con letrinas sistemas de agua, se aislaron bacterias, hongos y parásitos como (1).

El año 2012 en Ghana se llevó a cabo un estudio de la flora interna y externa de 61 cucarachas (Periplaneta americana), en un hospital de tercer nivel donde se evaluó frecuencia de microrganismos patógenos y perfiles de resistencia a los antibióticos. Se observa $19.7 \%$ presencia de rotavirus en la superficie del insecto, 4 tipos de parásitos, 8 bacterias nosocomiales, siendo la más prevalente la Klebsiella pneumoniae $29.5 \%$ en la parte interna y 26.2 externamente. Entre las bacterias resistentes a múltiples drogas oscila en 13.8\% Escherichia coli y $41.1 \%$ Klebsiella pneumoniae (10).

En nuestro país no existe investigación publicadarelacionada al tema que se ha investigado.Por tanto la finalidad de este estudio fue determinar el perfil de susceptibilidad y el rango de resistencia de bacterias colonizantes aisladas en cucarachas de un Hospital de Lima Metropolitana.

\section{Material y métodos}

Se recolectaron 100 cucarachas de distintas áreas de un Hospital de Lima Metropolitano en los meses de Junio y Julio del 2015, se aplicó el muestreo probabilístico de tipo conglomerado. 


\section{Captura de las cucarachas}

Se preparó una trampa doméstica con cinta adhesiva para recolectar las cucarachas.Se capturaron las cucarachas vivos de la especie (Blatella germánica), de las diferentes áreas dentro del hospital en un frasco estéril rotulado respectivamente.

\section{Aislamiento de bacterias}

El aislamiento de las bacterias que colonizan en la parte externa de la cucaracha, se realizaron por lavado con solución salina, tras la recogida, se inmovilizaron a las cucarachas de cuatro en cuatro a $4^{\circ} \mathrm{C}$ durante 10 a 20 minutos, luego inmerso en solución salina al 0,9\% fueron homogeneizados durante 1 minuto, se concentraron las muestras por centrifugación a 5000 RPM durante 10 minutos, del pellet, inicialmente se realizaron coloración de Gram y Ziehl Neelsen y luego se sembraron en medios selectivos adecuados Agar Sangre y Agar Maconkey (AS y MK,), luego se incubaron durante 24 a 48 horas a $37^{\circ} \mathrm{C}$.

\section{Tipificación de bacterias}

La determinación de género y especie se realizaron por métodos manuales convencionales utilizando medios diferenciales.Cada tipo de colonia se aislaron en los medios diferenciales tales como: TSI, LIA, CITRATO, UREA y SIM y se incubaron durante 24 horas a $37^{\circ} \mathrm{C}$. Luego, se observó la reacción bioquímica de las bacterias.

\section{Perfil de susceptibilidad empleando discos de difusión}

La prueba de susceptibilidad se realizó por método de difusión o Kirby Bauer.El Inóculo bacteriano se preparó en solución salina al 0.9\% a una concentración 0.05 según escala propuesto por MacFarland, los inóculos fueron sembrados en agar Müller Hinton, con un hisopo estéril.Los discos para Gram positivos (Ampicilina, Oxacilina, Eritromicina, Clindamicina, Vancomicina y cefazolina) y para Gram negativos (Ciprofloxacino, Trimetroprim + sulfametoxazol, Amikacina, Ceftriaxona, Imipenem y Amoxicilina + Ac. Clavulánico),fueron distribuidos de manera equidistante y las placas se incubaron a una

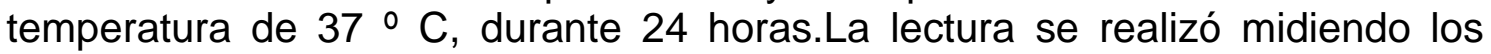
halos con una regla, comparando con las tablas desarrolladas por NCCLS.

Los datos fueron analizados mediante el programa estadístico SPSS versión 21.0 se determinaron medidas de tendencia central, medidas de dispersión, se emplearon tablas de frecuencia, de contingencia y porcentuales.

\section{Resultados}

Los resultados estadísticos que a continuación se detallan, corresponden a la evaluación respecto a la susceptibilidad y el rango de resistencia de las bacterias colonizantes aisladas, en cucarachas de las diferentes áreas de un Hospital de Lima Metropolitana. La muestra, recolectada mediante trampa 
doméstica con cinta adhesiva, estuvo formada por 100 cucarachas de la especie Blatella germánica.

\section{Áreas de recolección de las cucarachas}

Tabla № 1: Áreas del HLM de recolección de la muestra

\begin{tabular}{l|r|r|r}
\hline \multicolumn{1}{c|}{ Áreas } & Frecuencia & Porcentaje & $\begin{array}{c}\text { Porcentaje } \\
\text { acumulado }\end{array}$ \\
\hline Jardines & 16 & 16,0 & 16,0 \\
Pasadizos de nefrología & 16 & 16,0 & 32,0 \\
Pasadizos de neumología & 12 & 12,0 & 44,0 \\
Sótano & 8 & 8,0 & 52,0 \\
Tópico de neumología & 12 & 12,0 & 64,0 \\
Tópico de medicina & 8 & 8,0 & 72,0 \\
Tópico de gastroenterología & 8 & 8,0 & 80,0 \\
Sala de pacientes de neumología & 8 & 8,0 & 88,0 \\
Cocina de neumología & 8 & 8,0 & 96,0 \\
Baño de pacientes de neumología & 4 & 4,0 & 100,0 \\
Total & 100 & 100,0 & \\
\hline
\end{tabular}

Fuente: Elaboración propia

se observa que las áreas del hospital de Lima metropolitana donde se efectuó la recolección de las cucarachas en los meses de Junio y Julio del 2015 es como sigue: En los jardines se recolectaron 16 cucarachas, en los pasadizos de nefrología16, en los pasadizos de neumología 12; en el Sótano 8, en Tópico de neumología12, en Tópico de medicina 8, en Tópico de gastroenterología8, en Tópico de Sala de pacientes de neumología8, en cocina de neumología 8 y en baño de pacientes de neumología 4 cucarachas. Tabla № 01.

\section{Frecuencia de tipos de microorganismos aislados según el área}

\begin{tabular}{l|c|c}
\hline \multicolumn{1}{c|}{ Áreas } & Microorganismos Gram(-) & Microorganismos Gram(+) \\
\hline Jardines & Serratia marcescens & - \\
Pasadizos de nefrología & Serratia marcescens & $\begin{array}{c}\text { Staphylococcus coagulasa } \\
\text { neginetobacter sp. }\end{array}$ \\
Pasadizos de neumología & Klebsiella pneumoniae & Streptococcus viridans \\
Sótano & Serratia marcescens & - \\
Tópico de neumología & Klebsiella oxytoca & \\
& Escherichia coli & - \\
Tópico de medicina & Klebsiella pneumoniae & \\
& Klebsiella pneumoniae & Streptococcus viridans
\end{tabular}




\begin{tabular}{l|c|c} 
'Tópico de gastroenterología & $\begin{array}{c}\text { Klebsiella pneumoniae } \\
\text { Pseudomona sp. } \\
\text { Sala de pacientes de neumología }\end{array}$ & $\begin{array}{c}\text { Streptococcus viridans } \\
\text { Klebsilla pneumoniae }\end{array}$ \\
Cocina de neumología & $\begin{array}{c}\text { Klebsiella pneumoniae } \\
\text { Baño de pacientes de neumología }\end{array}$ & - \\
\hline
\end{tabular}

Tabla № 2:Frecuencia de tipos de microorganismos aisladas según las áreas del HLM

Fuente: Elaboración Propia

La frecuencia de tipos de microorganismos según el área de recolección, en las muestras de los jardines se aisló una sola especie de bacterias Gramnegativos (Serratia marcescens); en la muestra de los pasadizos de Nefrología se aislaron dos especies de bacterias gramnegativos (Serratia marcescens, Acinetobacter sp.) y Grampositivos (Staphylococcus coagulasa negativo); en la muestra de los pasadizos de Neumología se aislaron una especie de bacterias gramnegativos (Klebsiella pneumoniae) y grampositivos (Streptococcus viridans); en la muestra del sótano se aisló una especie de bacteria gramnegativos (Serratia marcescens); en la muestra del tópico de Neumología se aislaron tres especies de bacterias gramnegativos (Klebsiella oxytoca, Escherichia coli y Klebsiella pneumoniae); en las muestras de tópico de Medicina se aislaron una especie de bacteria gramnegativos (Klebsiella pneumoniae) y grampositivos (Streptococcus viridans); en las muestras de tópico de Gastroenterología se aislaron una especie de gramnegativos (Klebsiella pneumoniae) y grampositivos (Streptococcus viridans); en la muestra de sala de pacientes de Neumología se aisló dos especies de gramnegativos (Pseudomona sp., Klebsiella pneumoniae), en la muestra de cocina de Neumología se aisló una especie de gramnegativos (Klebsiella pneumoniae); y en la muestra de baño de pacientes de Neumología se aislaron una especie de gramnegativos (Klebsiella pneumoniae) y una especie de grampositivos (Streptococcus viridans)Tabla № 02.

\section{Coloración Gram de la muestra}

Tabla № 3:Gérmenes encontrados en la muestra

\begin{tabular}{l|r|r|r}
\hline & Frecuencia & Porcentaje & $\begin{array}{c}\text { Porcentaje } \\
\text { acumulado }\end{array}$ \\
\hline No se observa gérmenes & 16 & 16,0 & 16,0 \\
BGN & 48 & 48,0 & 64,0 \\
BGN y levaduras & 32 & 32,0 & 96,0 \\
CGP y levaduras & 4 & 4,0 & 100,0 \\
Total & 100 & 100,0 & \\
\hline
\end{tabular}

Fuente:Elaboración Propia

La coloración de Gram realizada a la muestra, formada por 100 cucarachas de la especie Blatella germánica. En 16 cucarachas de la muestra no se observaron gérmenes; en 48 cucarachas se observaron Bacilos Gram Negativos (BGN); en 32 cucarachas se observaron BGN y levaduras y solo en 
4 se observó Cocos Gram Positivos (CGP) y levadurasTabla № 03y los resultados de la tinción de Ziehl- Neelzen, para Micobacterium tuberculosisse obtuvo negativo;.

\section{Identificación de los gérmenes encontrados: Bacilos Gram- negativos}

Tabla № 5: Tipo de BGN encontrados en la muestra

\begin{tabular}{l|r|r|l}
\hline & Frecuencia & Porcentaje & Gérmen encontrado \\
\hline Cucarachas & 24 & 24,0 & Serratia marcescens \\
\hline & & & \\
& 46 & 4,0 & Acinetobacter sp. \\
& 36,0 & Klebsiella \\
& 4 & & pneumoniae \\
& 4,0 & Klebsiella oxytoca \\
& 4,0 & Scherichia coli \\
& 20 & 4,0 & Pseudomona sp. \\
& 100 & 20,0 & Ninguno \\
Total & 100,0 & \\
\hline
\end{tabular}

Fuente: Elaboración Propia

Tabla № 6: Tipo de CGP encontrados en la muestra

\begin{tabular}{l|r|r|l}
\hline & Frecuencia & Porcentaje & \multicolumn{1}{|c}{ Germen encontrado } \\
\hline Cucarachas & 4 & 4,0 & Staphylococcus coagulasa negativa \\
& 16 & 16,0 & Streptococcus viridans \\
& 80 & 80,0 & Ninguno \\
Total & 100 & 100,0 & \\
\hline
\end{tabular}

Fuente: Elaboración Propia

En 24 cucarachas se encontraron bacteriasSerratiamarcescens; en 4 cucarachas se encontraron Acinetobactersp; en 36 cucarachas se encontraron Klebsiellapneumoniae; en 4 cucarachas se encontraron laKlebsiellaoxytoca; en 8 cucarachas se encontraron Escherichiacoli; en 4 cucarachas se encontraron Pseudomona sp.Y en 20 cucarachas no se encontraron ningún microorganismo Tabla № 05y en 4 cucarachas se encontraron la especies de Staphylococcus coagulasa - negativa; en 16 cucarachas se encontraron bacteriasStreptococcus viridans y en 80 cucarachas no se encontraron ningún gérmen Coco Gram positivo. Tabla № 06 


\section{Antibiograma y sensibilidad de los Bacilos Gram Negativos}

\section{Ampicilina}

Tabla № 7: Antibiograma y sensibilidad de los BGN a la ampicilina

\begin{tabular}{l|r|r|r|r}
\hline & \multicolumn{4}{|c}{ Ampicilina } \\
\cline { 2 - 5 } & Sensible & Intermedio & Resistente & $\begin{array}{c}\text { No se } \\
\text { uso }\end{array}$ \\
\hline Serratia marcescens & - & - & - & $\mathrm{x}$ \\
Acinetobacter sp & - & - & - & $\mathrm{x}$ \\
KGN $\begin{array}{l}\text { Klebsiella pneumoniae } \\
\text { Klebsiella oxytoca }\end{array}$ & $\mathrm{S}$ & $\mathrm{I}$ & $\mathrm{R}$ & - \\
Scherichia coli & - & - & $\mathrm{R}$ & - \\
Pseudomona sp & - & - & $\mathrm{R}$ & - \\
\hline
\end{tabular}

Fuente: Elaboración Propia

El Antibiograma y sensibilidad a la Ampicilina de los Bacilos Gram Negativos (BGN), encontrados en la muestra. No se usó la ampicilina para los gérmenesSerratiamarcescens y Acinetobactersp, La Klebsiella pneumoniae hallada en un grupo de la muestra, fue sensible a la ampicilina, mientras que las encontradas en un segundo grupo fue intermedio y las encontradas en un tercer grupo fueron resistentes. Los gérmenes, Klebsiellaoxytoca, scherichiacoli yPseudomona sp. Fueron resistentes a la ampicilinaTabla № 07.

\section{Imipenem}

Tabla № 8: Antibiograma y sensibilidad de los BGN al Imipenem

\begin{tabular}{|l|r|r|r|r}
\hline & \multicolumn{5}{|c}{ Imipenem } \\
\cline { 2 - 5 } & Sensible & Intermedio & Resistente & $\begin{array}{c}\text { No se } \\
\text { uso }\end{array}$ \\
\hline \multirow{2}{*}{ Serratia marcescens } & $\mathrm{S}$ & - & - & - \\
BGN & $\mathrm{S}$ & - & - & - \\
Kcinetobacter sp & $\mathrm{S}$ & $\mathrm{I}$ & - & - \\
Klebsiella pneumoniae & $\mathrm{S}$ & - & - & - \\
\hline
\end{tabular}




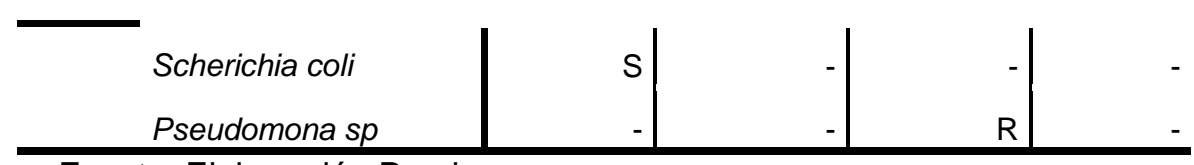

Fuente: Elaboración Propia

El Antibiograma y Sensibilidad allmipenem de los BGN, encontrados en la muestra. La bacteria Serratiamarcescens y elAcinetobactersp, fueron sensibles al Imipenem; LaKlebsiellapneumoniae hallada en un grupo de la muestra fue sensible, mientras que las encontradas en un segundo grupo fue intermedio. Los gérmenes, Klebsiellaoxytocay Escherichiacoli fueron sensibles y la Pseudomona sp.Resistente al mismotabla № 8.

\section{Amikacina}

Tabla № 9: Antibiograma y sensibilidad de los BGN a la Amikacina

\begin{tabular}{l|r|r|r|r}
\hline & \multicolumn{4}{|c}{ Amikacina } \\
\cline { 2 - 5 } & Sensible & Intermedio & Resistente & $\begin{array}{c}\text { No se } \\
\text { uso }\end{array}$ \\
\hline Serratia marcescens & $S$ & - & - & - \\
Acinetobacter sp & S & - & - & - \\
BGN $\begin{array}{l}\text { Klebsiella pneumoniae } \\
\text { Klebsiella oxytoca }\end{array}$ & - & - & - & $\mathrm{x}$ \\
Escherichia coli & - & - & - & $\mathrm{x}$ \\
Pseudomona sp & - & - & - & $\mathrm{x}$ \\
\hline
\end{tabular}

Fuente: Elaboración Propia

El Antibiograma y sensibilidad a la Amikacina de los Bacilos Gram Negativos (BGN), solo se usó para las cepas,Serratiamarcescens y Acinetobactersp, donde fueron sensibles a la AmikacinaTabla № 9.

\section{Ciprofloxacino}

Tabla № 10: Antibiograma y sensibilidad de los BGN a la Ciprofloxacino

\begin{tabular}{|c|c|c|c|c|c|}
\hline & & \multicolumn{3}{|c|}{ Ciprofloxacino } & \multirow[b]{2}{*}{$\begin{array}{c}\text { No se } \\
\text { uso }\end{array}$} \\
\hline & & Sensible & Intermedio & Resistente & \\
\hline \multirow{6}{*}{ BGN } & Serratia marcescens & $S$ & - & - & - \\
\hline & Acinetobacter sp & $S$ & - & - & - \\
\hline & Klebsiella pneumoniae & $\mathrm{S}$ & - & - & - \\
\hline & Klebsiella oxytoca & - & 1 & - & - \\
\hline & Escherichia coli & - & 1 & - & - \\
\hline & Pseudomona sp & $\mathrm{S}$ & - & - & - \\
\hline
\end{tabular}

Fuente: Elaboración Propia 
EL Antibiograma y sensibilidad a la Ciprofloxacinolos gérmenesSerratiamarcescens,Acinetobactersp, y Klebsiella pneumoniae fueron sensibles alCiprofloxacino yKlebsiellaoxytocay Escherichiacoli presentaron una resistencia intermedia y el germenPseudomona sp. Fuesensible al mismo Tabla № 10.

\section{Sulfametoxazol + trimetoprim}

Tabla № 11: Antibiograma y sensibilidad de los BGN al Sulfametoxazol + trimetoprim

\begin{tabular}{l|r|r|r|r}
\hline & \multicolumn{4}{|c}{ Sulfametoxazol + trimetoprim } \\
\cline { 2 - 5 } & Sensible & Intermedio & Resistente & $\begin{array}{c}\text { No se } \\
\text { uso }\end{array}$ \\
\hline Serratia marcescens & $\mathrm{S}$ & - & - & - \\
Acinetobacter sp & $\mathrm{S}$ & - & - & - \\
BGN Klebsiella pneumoniae & $\mathrm{S}$ & - & - & - \\
Klebsiella oxytoca & - & - & $\mathrm{R}$ & - \\
Scherichia coli & - & - & $\mathrm{R}$ & - \\
Pseudomona sp & $\mathrm{S}$ & - & - & - \\
\hline
\end{tabular}

Fuente: Elaboración Propia

El Antibiograma y sensibilidad alSulfametoxazol + trimetoprim paraSerratiamarcescens, Acinetobactersp,Pseudomona sp. yKlebsiella pneumoniae fueron sensibles, KlebsiellaoxytocayEscherichiacoli fueron resistentes alSulfametoxazol + trimetoprimTabla № 11.

\section{Amoxicilina + Ac. Clavulánico}

Tabla № 12: Antibiograma y sensibilidad de los BGN a la Amoxicilina + Acido Clavulánico

\begin{tabular}{l|r|r|r|r}
\hline & \multicolumn{4}{|c}{ Amoxicilina + Acido Clavulánico } \\
\cline { 2 - 5 } & Sensible & Intermedio & Resistente & $\begin{array}{c}\text { No se } \\
\text { uso }\end{array}$ \\
\hline Serratia marcescens & $\mathrm{S}$ & - & - & - \\
Acinetobacter sp & $\mathrm{S}$ & - & - & - \\
BGN Klebsiella pneumoniae & $\mathrm{S}$ & - & - & - \\
Klebsiella oxytoca & - & - & $\mathrm{R}$ & - \\
Scherichia coli & $\mathrm{S}$ & - & - & - \\
\hline
\end{tabular}

Fuente: Elaboración Propia 
El Antibiograma y sensibilidad a la Amoxicilina + Acido Clavulánico paralas bacteriasSerratiamarcescens, Acinetobacter $s p$, y Klebsiella pneumoniae, Escherichiacoli yPseudomona $s p$ fueron sensibles y resistente para KlebsiellaoxytocaTabla № 12.

\section{Ceftriaxona}

Tabla № 13: Antibiograma y sensibilidad de los BGN a la Ceftriaxona

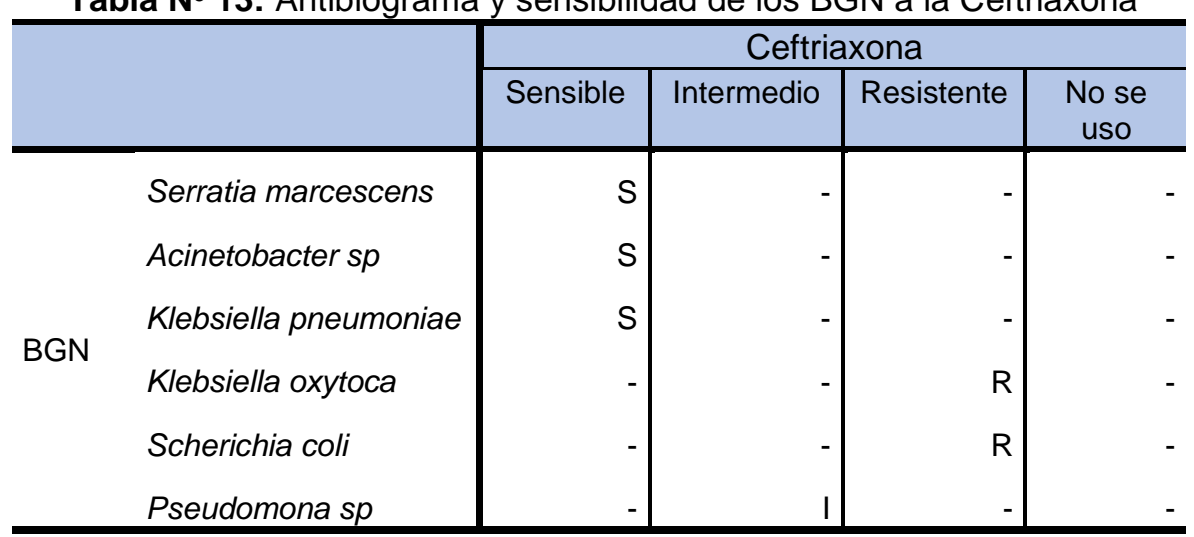

Fuente: Elaboración Propia

El Antibiograma y sensibilidad a la Ceftriaxonapara los gérmenes Serratiamarcescens, Acinetobacter sp, y Klebsiellapneumoniae fueron sensibles, para Klebsiellaoxytoca y Escherichia coli presentaron resistencia e intermedia paraPseudomona sp. Tabla № 13.

\section{Antibiograma y Sensibilidad de los Cocos Gram Positivos (CGP)}

\section{Clindamicina}

Tabla № 14:Antibiograma y Sensibilidad de los CGP a la Clindamicina

\begin{tabular}{ll|r|r|r|r}
\hline & \multicolumn{5}{|c}{ Clindamicina } \\
\cline { 2 - 5 } & Sensible & Intermedio & Resistente & $\begin{array}{c}\text { No se } \\
\text { uso }\end{array}$ \\
\hline \multirow{2}{*}{$\begin{array}{l}\text { Staphylococcus } \\
\text { Cop } \quad \begin{array}{l}\text { Coagulasa negativa } \\
\text { Streptococcus viridans }\end{array}\end{array}$} & $\mathrm{S}$ & - & - & \\
\hline
\end{tabular}

Fuente: Elaboración Propia

El Antibiograma y Sensibilidad a la Clindamicina de los Cocos Gram Positivos (CGP), encontrados en la muestra. El gérmen Staphylococcus coagulasa negativa fue sensible, mientras que el Streptococcus viridans., encontradas en 
un grupo de la muestra fue intermedio y en un segundo grupo resistenteTabla № 14.

\section{Eritromicina}

Tabla № 15: Antibiograma y sensibilidad de los CGP a la Eritromicina

\begin{tabular}{|c|c|c|c|c|c|}
\hline & & \multicolumn{4}{|c|}{ Eritromicina } \\
\hline & & Sensible & Intermedio & Resistente & $\begin{array}{c}\text { No se } \\
\text { uso }\end{array}$ \\
\hline \multirow{3}{*}{ CGP } & Staphylococcus & $\mathrm{x}$ & - & - & - \\
\hline & coagulasa negativa & & & & \\
\hline & Streptococcus viridans & $\mathrm{x}$ & $x$ & $\mathrm{x}$ & - \\
\hline
\end{tabular}

Fuente: Elaboración Propia

El Antibiograma y sensibilidad a la Eritromicina paraStaphylococcus coagulasa - negativa fue sensible, paraStreptococcus viridans., encontrados en un grupo de la muestra, fue sensible; en un segundo grupo fue intermedio y en un tercer grupo resistenteTabla № 15.

\section{Oxacilina}

Tabla № 16: Antibiograma y sensibilidad de los CGP a la Oxicilina

\begin{tabular}{lr|r|r|r|r}
\hline & \multicolumn{4}{|c}{ Oxicilina } \\
\cline { 2 - 5 } & Sensible & Intermedio & Resistente & $\begin{array}{c}\text { No se } \\
\text { uso }\end{array}$ \\
\hline Staphylococcus & $\mathrm{S}$ & - & - & - \\
CGP $\quad \begin{array}{l}\text { coagulasa negativa } \\
\text { Streptococcus viridans }\end{array}$ & $\mathrm{S}$ & $\mathrm{I}$ & $\mathrm{R}$ & - \\
\hline
\end{tabular}

Fuente: Elaboración Propia

El Antibiograma y sensibilidad a la OxacilinaparaStaphylococcus coagulasa negativa fue sensible, mientras paraStreptococcus viridans., encontrados en un grupo de la muestra fue sensible, en un segundo grupo fue de intermedio y en un tercer grupo fue resistente Tabla № 16.

\section{Cefazolina}

Tabla № 17: Antibiograma y sensibilidad de los CGP a la Cefazolina 


\begin{tabular}{|c|c|c|c|c|c|}
\hline & & \multicolumn{4}{|c|}{ Cefazolina } \\
\hline & & Sensible & Intermedio & Resistente & $\begin{array}{c}\text { No se } \\
\text { uso }\end{array}$ \\
\hline \multirow{3}{*}{ CGP } & Staphylococcus & $S$ & - & - & - \\
\hline & coagulasa negativa & & & & \\
\hline & Streptococcus viridans & $S$ & - & $\mathrm{R}$ & - \\
\hline
\end{tabular}

Fuente: Elaboración Propia

El Antibiograma y sensibilidad a la Cefazolina paraStaphylococcus coagulasa negativa fue sensible y paraStreptococcus viridans., de unprimer grupo de muestra fue sensible y en un segundo grupo fue resistente Tabla № 17.

\section{Ciprofloxacino}

Tabla № 18: Antibiograma y sensibilidad de los CGP al Ciprofloxacino

\begin{tabular}{|c|c|c|c|c|c|}
\hline & & \multicolumn{4}{|c|}{ Ciprofloxacino } \\
\hline & & Sensible & Intermedio & Resistente & $\begin{array}{c}\text { No se } \\
\text { uso }\end{array}$ \\
\hline \multirow{3}{*}{ CGP } & Staphylococcus & S & & & \\
\hline & coagulasa negativa & & & & \\
\hline & Streptococcus viridans & $\mathrm{S}$ & & & \\
\hline
\end{tabular}

Fuente: Elaboración Propia

El Antibiograma y sensibilidad alCiprofloxacinoparaStaphylococcus coagulasa negativa y Streptococcus viridans fueron sensiblestabla № 18.

\section{Vancomicina}

Tabla № 19: Antibiograma y sensibilidad de los CGP al Vancomicina

\begin{tabular}{ll|r|r|r|r}
\hline & \multicolumn{3}{|c}{ Vancomicina } \\
\cline { 2 - 5 } & Sensible & Intermedio & Resistente & $\begin{array}{c}\text { No se } \\
\text { uso }\end{array}$ \\
\hline \multirow{2}{*}{$\begin{array}{l}\text { Staphylococcus } \\
\text { coagulasa negativa }\end{array}$} & $\mathrm{S}$ & - & - & - \\
& & & & & \\
\hline
\end{tabular}

Fuente: Elaboración Propia

El Antibiograma y sensibilidad al VancomicinaparaStaphylococcus coagulasa negativa y Streptococcus viridans fueron sensiblestabla № 19.

\section{Discusion}

La recolección de las cien cucarachas de la especie Blatella germánica se llevó a cabo en un Hospital de cuarto nivel de Lima metropolitano(12). En el presente estudio realizado de cada muestra sembrada se aislaron de 0 2microorganismos patógenos; Sin embargoel año 2002 se realizó un 
estudio de perfil de resistencia antimicrobiana de 103 cucarachas Periplaneta americano, en una institución de salud en Brasil. Los insectos se recolectaron de cinco unidades diferentes de la fuente: de la cual se obtuvieron 126 aislamientos; $71.4 \%$ tenían especies de la familia Enterobacteriaceae, $25.4 \%$ estafilococos coagulasa -negativo, 3.2\% fueron bacilos Gram-negativos, levaduras y hongos filamentosos en $97 \%$ de las cucarachas, y el número promedio de los tipos de microorganismos aislados por cucaracha varió de uno a tres (11). La búsqueda de microrganismos altamente patógenos y multidrogoresistentes que colonizan en las cucarachas nunca han sido estudiados en nuestro país; sin embargo en el presente estudio hemos encontrado bacterias Grampositivos(Staphylococcuscoagulasa- negativa4\% y Streptococcusviridans 16\%),ybacterias Gramnegativos (Serratiamarcenses 24\%, Acinetobactersp 4\%,Klebsiellapneumoniae 36\%,Klebsiellaoxytoca 4\%,Escherichiacoli 8\%yPseudomonasp 4\%; sin embargo en la coloración de ZiehINeelsen no se encontró Micobacterium tuberculosis. La Blatella germánica es un vector potencial de gérmenes patógenos en los hospitales, es así que en Taiwán en el año 2004 se hizo un estudio de dos especies (Blatella germánica y Periplaneta americano) debido a la infestación en más de $40 \%$ de los hospitales y las bacterias que se aislaron casi todas eran multidrogorresistentes (2).En el presente estudio de los 06 especies de bacteriasGramnegativosaislados Klebsiella pneumoniae y Serratia marcescens han sido los más prevalentesy en un estudio realizado en el año 2012 en Ghana se llevó a cabo un estudio de la flora interna y externa de 61 cucarachas (Periplaneta americana), en un hospital de tercer nivel donde se evaluó frecuencia de microrganismos patógenos y perfiles de resistencia a los antibióticos. Siendo la más prevalente la Klebsiella pneumoniae que ocurrió internamente en $29.5 \%$ y 26.2 externamente. Entre las bacterias resistentes a múltiples drogas oscila en $13.8 \%$ (Escherichia coli) y $41.1 \%$ (Klebsiella pneumoniae) (10).En el presente estudio de los 08 especies de bacteriasGramnegativos $y \quad$ Grampositivas aisladas, Klebsiella oxytoca,Escherichiacoli y Streptococcus viridans,presentaron resistencia a la mayoría de los antimicrobianos, lo cual difiere un estudio realizado en Taiwán en el año 2004 a dos especies (Blatella germánica y Periplaneta americano) debido a la infestación en más de $40 \%$ de los hospitales y las bacterias que se aislaron casi todas eran multidrogorresistentes (2)

Agradecimiento:

Al Dr. Alfonso Martin Cabello Vílchez, por sus enseñanzas, dedicación y Ayuda en la realización del presente trabajo.

Al Lic. Jorge Fernández Baldeón, Por la asesoría metodológica para el desarrollode este trabajo.

A la Lic. Yanina Soto Agreda por la orientacióny dedicación en el desarrollo de esta investigación.

Al Director de la Escuela deFarmacia y Bioquímica y Nutrición de la UAPDr. Javier Gómez Guerreiro porlas Facilidades prestadas para el desarrollo de esta Investigación. 


\section{Referencia bibliografica}

1. Y.M. Tatfenga, M.U. Usuanleleb, A. Orukpeb, A.K. Digbana, M. Okoduac,F. Oviasogie, et al. Mechanical transmission of pathogenic organisms: the role ofcockroaches. J Vect Borne. 2005; 42; 129 - 134.

2. HsiuH. P., Wei-Ch. Ch., ChienFP; Source. Cockroaches as Potential Vectors of Nosocomial Infections. Chicago journals. 2004; 25(11); 979 984.

3. P.M.Hawkey. Thegrowingburdenofantimicrobialresistance. JournalofAntimicrobialChemotherapy. 2008; 62 (1); 1 - 9 .

4. Lebrun G, Chadda $K$, Reboux AH, $r$ Martinet $O$, Gaüzère BA, Cockroaches (Ectobiusvittiventris) in an Intensive Care Unit. Emerging Infectious Disease. 2009; 15(3); DOI: 10.3201/eid1503.071482.

5. Fotedar R, Banerjee U,Verma A. Cockroaches (Blattellagermánica) as carriers ofmicroorganismsofmedical importance inhospitals. DepartmentsofMicrobiologyand Surgery,AlllndialnstituteofMedical Sciences. 1991; 107; $181-187$.

6. Bonomo RA. MultipleAntibiotic-ResistantBacteriainLong-TermCareFacilities:AnEmergingProbleminthePracticeofInfectiousDiseasesClini cal Infectious Diseases. 2000; 31:1414-22.

7. Mojtaba L., BehrozD. Seyed H.M.-K. Toxicity of Pyrethroid and Organophosphorous InsecticidesagainstTwo Field Collected Strains of the German CockroachBlattellagermánica (Blattaria: Blattellidae).J Arthropod-Borne. 2012; 6(2); 112 - 118.

8. Nitat $\mathrm{S}$, Wanpen Ch. A revisit to cockroach allergens Asian Pac J Allergy Immunol. 2010; 28: 95-106.

9. lannacone J, AlvariñoL: Integración del control químico y etológico para la supresión poblacional deBlatella germánica (Linnaeus)(Dictyoptera: Blatellidae) en Lima, Perú: ParasitolLatinoam: 2007. 62: 7 - 15.

10. Tetteh-QP, Donkor ES, Attah SK, Duedu KO, Afutu E, Boamah I, et. Al.Microbial carriage of cockroaches at a tertiary care hospital in ghana: Department of Microbiology, University of Ghana Medical School. 2013: 7:59-66. 
11. Aparecida PM, Gir E, Severino PM, Reis C, Pimenta FC; Profile of antimicrobial resistance of bacteria isolated from cockroaches (Periplanetaamericana) in a Brazilian health care institution; Brazilian Journal of Infectious Diseases. 2006; 10(1); 26-32.

12. http://www.essalud.gob.pe/nuestras-redes-asistenciales/la-victoria/.

13. Díaz PC, Álvarez GY, De Armas RY y Bisset LJA; Determinación de la resistencia a insecticidas y mecanismos de resistencia en cepas de Blattellagermanica (Dictyoptera: Blattellidae);Revista Cubana de Medicina Tropical;2007; 59 (2);159-65.

14. Maketon M, Haominchan A; Hotaka D; Control de la cucaracha americana (Periplaneta americana) y de la cucaracha alemana (Blattellagermanica) por nematodos entomopatógenos; 2010; Revista Colombiana de Entomología 36 (2); 249-253.

15. Fernández F, Padola NL; Escherichiacoliverocitotoxigénico: varias cuestiones y los tambos también; Revista Argentina de Microbiología; 2012; 44; 312-323.

16. Bisset JA; Uso correcto de insecticidas: control de la resistencia; Revista Cubana de Medicina Tropical; Uso correcto de insecticidas: control de la resistencia; 2002,54(3); 202-219.

17. Mendoza AA, Mansilla CG; Rinitis alérgica; Revista. Boliviana de Pediatría; 2002; 41 (1); 50-53.

18. Abdo RA, Cué BM, Álvarez CM; Asma bronquial: factores de riesgo de las crisis y factores preventivos; Revista Cubana Medicina General Integral; 2007; 23(3); 11-29.

19. Valdivia CG; Asma bronquial y enfermedades atópicas como problema emergente de Salud Pública: nuevas hipótesis etiológicas. La experiencia de sociedades desarrolladas. Revista médica de Chile; 2000; 128(3); 339-346.

20. Arruda CH.E; Pruebas diagnósticas en alergia y su utilidad clínica; Revista Médica Herediana; 2004; 15(2); 113-117.

21. Echevarría ZJ; Resistencia bacteriana; Revista Médica Herediana; 1998; $9(2)$ 53-55. 
22. Fernández RF, López HJ, Ponce MLM, Machado BC; Resistencia bacteriana; Revista Cubana Medicina Militar; 2003, 32(1); 44 - 8.

23. Quiñones PD;Resistencia antimicrobiana en aislamientos clínicos de Klebsiella spp. y producción de B-lactamasas de espectro extendido en hospitales de Cuba. Revista Cubana Medicina Tropical; 2014; 66(3); 386399.

24. Merino LA;Pseudomonas aeruginosa: una bacteria con personalidades múltiples. Revista Argentina deMicrobiología; 2007; 39(3); 143-143.

25.Palavecino RE; Estreptococos grupo anginosus: ¿Es su identificación clínicamente importante?; Revista Chilena de Infectología; 2004; 21(3); 261-267.

26. Vecchiola HM; Infecciones por Acinetobacter; Revista Chilena Infectologia; 2008; 25(5); 397-399.

27. Silva OF;Serratia marcescens; Revista Chilena de Infectología; 2010; 27(3); 209-210.

Email: S.decanatouap@gmail.com

TL. +51987220183 